\title{
Beitrag zur Frage der Schlammeindickung
}

Bericht über die rom Tiefbauamt der Stadt Zürich in den Fabren 1950 und 1951 durchgeführten Schlammeindickungspersuche

Von Dipl.-Ing. A. HörLER

Chef der technischen Abteilung der EAWAG

Mitteilung Nr. 133 der Eidg. Anstalt für Wasserversorgung, Abwasserreinigung und Gewässerschutz der ETH, Zürich. Direktor: Prof.Dr. O. JAAG

Manuskript eingegangen am 7. Oktober 1957

\section{Zweck der Versuche}

Bei der Projektierung von Abwasserreinigungsanlagen stellt sich immer wieder die Frage nach der zweckmässigsten Schlammbeseitigung. Neben dem Frischschlamm aus den Absetzbecken wird heute in der Regel auch der überschüssige Schlamm aus dem biologischen Teil der Abwasserreinigungsanlagen in Faulräumen ausgefault. Das Volumen des Überschußschlammes aus den Belebtschlammanlagen beträgt oft ein Vielfaches desjenigen des Frischschlammes. Um nicht unwirtschaftlich grosse Faulräume erstellen zu müssen, wird der Überschußschlamm nicht direkt von den Nachklärbecken in die Faulräume verfrachtet, sondern vorerst auf irgendeine Weise eingedickt. Dies kann nach dem von IMHOFF vorgeschlagenen Verfahren erfolgen durch Einleitung des Überschußschlammes in den Zulauf zum Absetzbecken. Dabei setzt sich der biologische Überschußschlamm gemeinsam mit dem Frischschlamm oder Primärschlamm im Absetzbecken ab und dickt sich dort beim Stehenlassen ein, oder es kann die Eindickung des Schlammes in eigenen Bauwerken erfolgen. Für diese Eindickungsvorrichtungen ist die Kenntnis der gesamten täglich zu behandelnden Schlammenge wesentlich, ferner die Eindickungsdauer, um feststellen zu können, ob täglich nur mit einem einschichtigen oder unter Umständen mit einem zwei- oder mehrschichtigen Eindickungsbetrieb gerechnet werden kann, sofern nicht eine Eindickung im Durchlaufbetrieb in Frage kommt. Die Grösse der Faulräume ist abhängig vom Mass der 
Eindickungsmöglichkeit des Schlammes. Dabei ist zum voraus nicht ohne weiteres klar, ob es zweckmässig ist, nur den äusserst voluminösen, wasserreichen Überschußschlamm der biologischen Reinigungsstufe für sich allein einzudicken, oder ob eine erfolgreichere Eindickung erhalten wird, wenn zuerst der Primärschlamm mit dem Überschußschlamm, evtl. dem Faulschlamm (ausgefaulter Schlamm), vermengt und die Schlammmischung eingedickt wird.

Das Tiefbauamt der Stadt Zürich hat vom November I950 bis Oktober 195I Versuche durchgeführt, die eine Abklärung der oben aufgeworfenen Fragen für die Verhältnisse der Stadt Zürich bezweckten. An Schlamm stand zur Verfügung:

Frischschlamm (Primärschlamm) aus der mechanischen Kläranlage Werdhölzli der Stadt Zürich, der entsprechend den in der Grob- und Feinklärung anfallenden Schlammengen verhältnisgleich gemischt wurde.

Überschußschlamm (Belebtschlamm) aus der Versuchsanlage der Eidgenössischen Anstalt für Wasserversorgung, Abwasserreinigung und Gewässerschutz an der ETH (EAWAG).

Faulschlamm aus den überlasteten Faulräumen der Kläranlage Werdhölzli (vorerst wird nur Primärschlamm ausgefault).

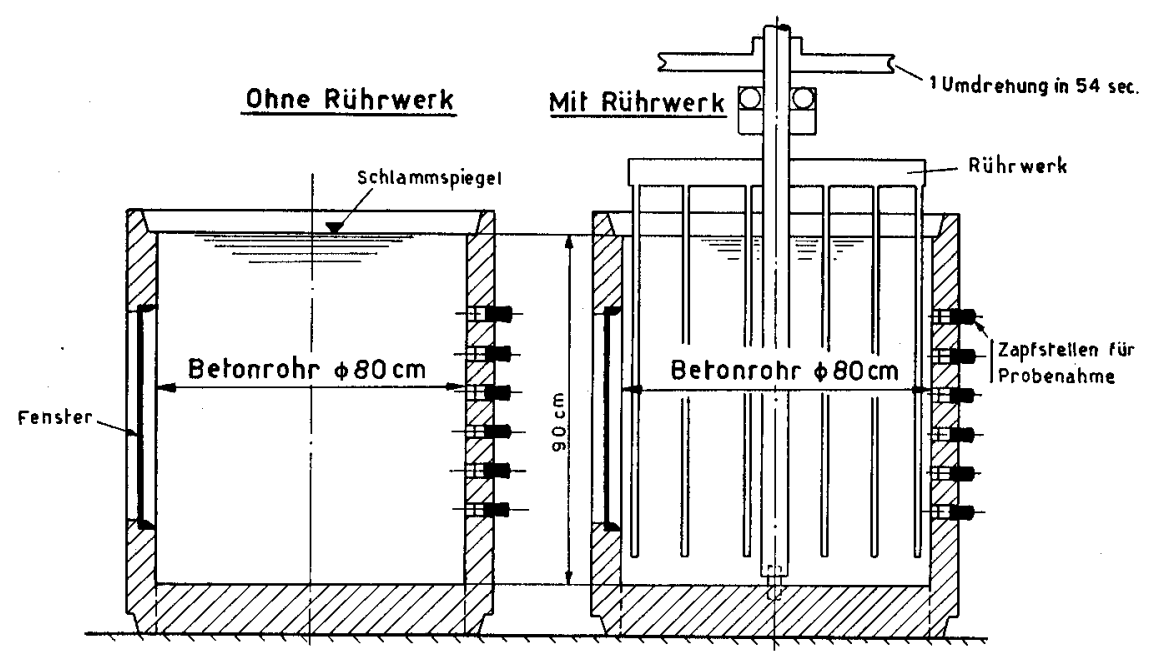

Abbildung 1

Versuchsanordnung. 


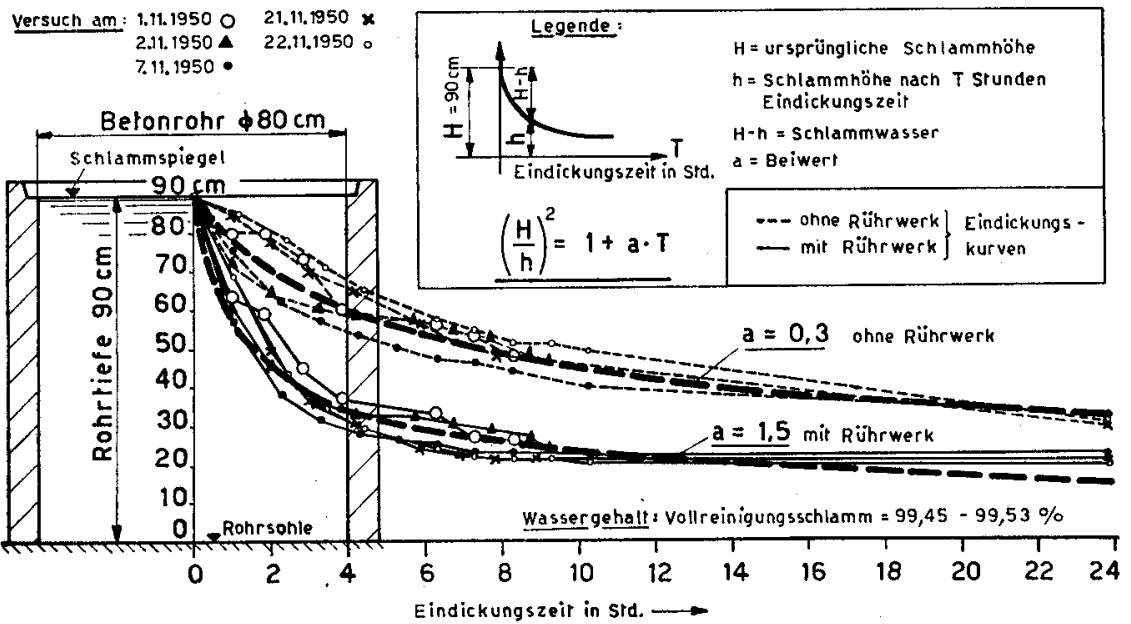

Abbildung 2

liindickung von Überschußschlamm aus biologischer Vollreinigungsanlage allein

(Versuche vom November 1950).

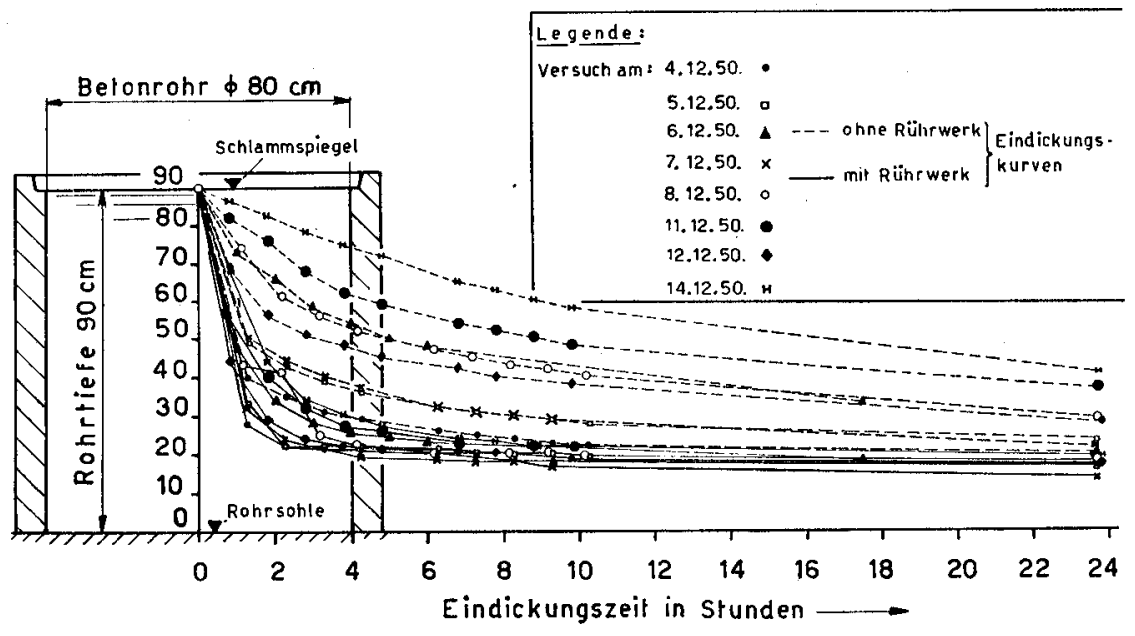

Wassergehalt [nicht eingedickt] $99,46-99,63 \%$

Abbildung 3

Eindickung von Überschußschlamm aus biologischer Vollreinigungsanlage allein

(Versuche vom Dezember 1950). 


\section{Versuchsanordnung}

Versuchsresultate wurden in zwei Richtungen angestrebt:

I. Durch Verwendung verschiedener Schlammischungen.

2. Durch Vergleich mit verschiedenen Eindickungsmassnahmen (mit und ohne Rührwerk, mit und ohne Zugabe von Chlor).

Aus diesem Grunde mussten Parallelversuche durchgeführt werden. Zwei Betonrohre von $80 \mathrm{~cm}$ Durchmesser mit Beobachtungsfenstern und Zapfstellen zum Ablassen des Schlammes wurden $90 \mathrm{~cm}$ tief mit jeweils gleichen Schlammischungen gefüllt und während 24 Stunden stehen gelassen (Abb.I). In einem der beiden Behälter wurde ein Rührwerk aus $2 \mathrm{~cm}$ starken Gasrohren eingebaut, das durch langsames Drehen (eine Umdrehung in 54 Sekunden) den Absetz- bzw. Eindickungsvorgang beschleunigte. Das Rührwerk arbeitete in der Regel ebenfalls während 24 Stunden.

\section{Versuchsprogramm und Versuchsresultate}

\section{Versuchsserie A}

Zur Eindickung gelangte Überschußschlamm aus einer biologischen Voll reinigungsanlage. Die Belastung der Belebtschlammanlage betrug nach Angaben der EAWAG $7 \mathrm{~m}^{3}$ Abwasser pro Kubikmeter Belüftungsraum und Tag, die Belüftungszeit somit 3,4 Stunden, der Reinigungseffekt, gemessen am stägigen biochemischen Sauerstoffbedarf, rund $90 \%$.

\section{r. Mit Belebtscblamm allein (Versucbsserien $A_{1} / 1+A_{1} / 2$ )}

Der Wassergehalt des Schlammes schwankte zwischen 99,45 und $99,53 \%$. Die beiden Behälter wurden mit je 4501 Überschußschlamm der Belebtschlammanlage gefüllt und je 5 Parallelversuche, ohne und mit Rührwerk, von 8-24 Stunden Eindickungszeit durchgeführt.

Die Versuchsresultate sind in den Abbildungen 2 und 3 dargestellt. Das Absinken des Schlammspiegels mit zunehmender Eindickungszeit ist aus den angegebenen Kurven deutlich erkennbar (Schlammtiefe bei Beginn der Versuche zur Zeit o stets $90 \mathrm{~cm}$ ). Ebenso geht hervor, dass mit dem Rührwerk eine bedeutend raschere und bei den verschiedenen Versuchen gleichmässigere Eindickung erzielt werden kann als durch blosses Stehenlassen des Schlammes allein.

Zur Kontrolle wurde auch der Wassergehalt des eingedickten Schlammes durch Analysen bestimmt. Der Vergleich mit dem aus den Beob- 
achtungszahlen errechneten Wassergehalt nach der Eindickung war wenig befriedigend. Da in der Praxis die über dem eingedickten Schlamm befindliche Wassersäule rein visuell festgestellt wird, hat man sich bei den letzten Versuchen mit der Messung der eingedickten Schichtdicke und mit der Berechnung des theoretischen Wassergehaltes des eingedickten Schlammes aus dem ursprünglichen Wassergehalt begnügt.

Wichtig dürfte die Feststellung sein, dass der Eindickungsvorgang mit Rührwerk praktisch in 4-6 Stunden vollendet war, während beim blossen Stehenlassen des Schlammes auch nach I2 Stunden Eindickungszeit noch eine deutliche Abnahme des Schlammvolumens festgestellt werden konnte. Bei der Anordnung mit Rührwerk war eine Eindickung auf etwa $1 / 3^{-1 / 4}$ der ursprünglichen Schlammenge innert 6 Stunden möglich, ohne Rührwerk in 6-12 Stunden nur etwa auf die Hälfte.

Der Eindickungsvorgang geht gesetzmässig vor sich. Aus Abbildung 2 ist ersichtlich, dass bei dieser Versuchsserie die Schlammeindickung als quadratische Funktion der Eindickungszeit dargestellt werden kann, und zwar sowohl mit als auch ohne Verwendung eines Rührwerkes. Aus den Versuchen vom I., 2., 7., 2I. und 22. November 1950 geht hervor, dass die Eindickungskurve angenähert folgender Gleichung gehorcht:

$$
\left(\frac{H}{h}\right)^{2}=1+a T,
$$

wobei $H$ ursprüngliche Stärke der Schlammschicht; $h$ Höhe der Schlammschicht nach $T$ Stunden Eindickungszeit; $a$ Beiwert.

Der Beiwert $a$ ist in erster Linie abhängig von der Eindickungsart. Für vorliegende Versuchsserie betrug $a$ bei blossem Stehenlassen des Schlammes 0,3 , bei Umrühren mit dem Rührwerk I, 5. Die entsprechenden theoretischen Kurven sind in der Abbildung 2 eingetragen.

Erwähnt sei noch, dass die Schlammeindickung den gleichen Gesetzen gehorcht wie die aus dem Erdbau bekannte Setzung von Gebäuden über Tonschichten.

Nach 41/4, 81/4 bzw. 233/4 Stunden Eindickungszeit wurde eine Eindickung auf folgende Werte in Prozent der ursprünglichen Schlammengen erzielt, wobei $\boldsymbol{s}^{\mathbf{1}}$ ) die Wurzel aus der Streuung

$$
s^{2}=\frac{1}{N-1} \sum_{i=1}^{N}\left(x_{i}-\bar{x}\right)^{2},
$$

bedeutet $\left(x_{i}\right.$ beobachtete Einzelwerte und $\bar{x}$ Durchschnitt der Einzelwerte).

1) Erklärung im Fuss der Tabelle 1. 
Tabelle 1

Eindickung auf Prozente des ursprünglicben Scblammpolumens

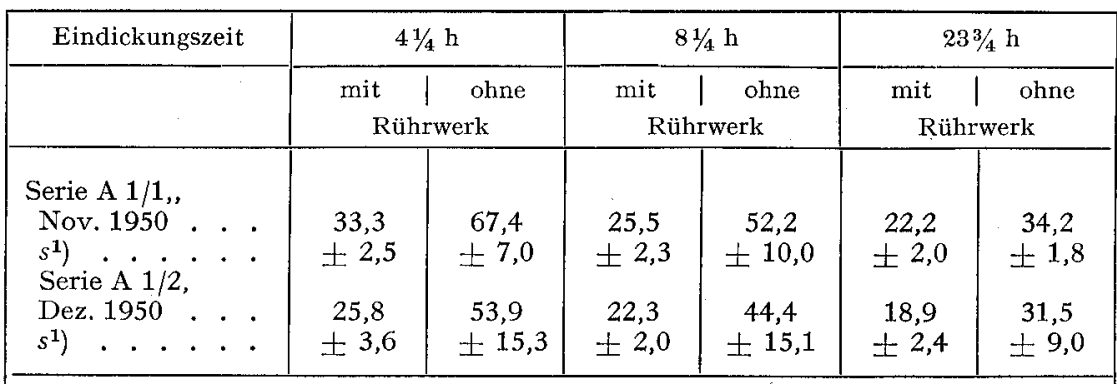

1) Die $s$-Werte in Prozent sind zu den Prozentzahlen des eingedickten Schlammes $z u$ addieren bzw. von ihnen zu subtrahieren.

Das Resultat dieser Versuchsreihen, die in den Abbildungen 2 und 3 dargestellt sind, kann wie folgt zusammengefasst werden:

Die Eindickung mit Rührwerk geht stets erheblich rascher vor sich als beim blossen Stehenlassen des Schlammes. Bis zu Eindickungszeiten von 8 Stunden kann bei Inbetriebnahme des Rührwerkes etwa eine doppelt so starke Eindickung erzielt werden als ohne Rührwerk. Für längere Eindickungszeiten nimmt der Unterschied ab. Weiter ist festzustellen, dass fast durchweg die Streuung der Versuchsresultate erheblich geringer ist beim Eindicken mit Rührwerk als ohne.

\section{Belebtscblamm aus Vollreinigung + primärer Scblamm}

a) Versuchsserie A2. Aus den Versuchen ohne Beimischung von Primärschlamm oder Frischschlamm aus der Vorreinigung ging hervor, dass sich Belebtschlamm aus der Vollreinigung allein von einem Wassergehalt von $99,5 \%$ nur auf etwa $97,5-98,5 \%$ Wassergehalt mit den beschriebenen Methoden eindicken liess. Es stellte sich deshalb die Frage, ob mit Beimischung von Frischschlamm zum Belebtschlamm günstigere Eindickungswerte erzielt werden können, weshalb in einer weiteren Versuchsserie (Abb.4) Parallelversuche mit und ohne Rührwerk auch unter Beimischung von Frischschlamm zum Belebtschlamm durchgeführt wurden.

Als Ergebnis dieser Versuche ist festzustellen, dass die Eindickung des Schlammes ganz allgemein rascher erfolgt ist als bei den Versuchsserien 


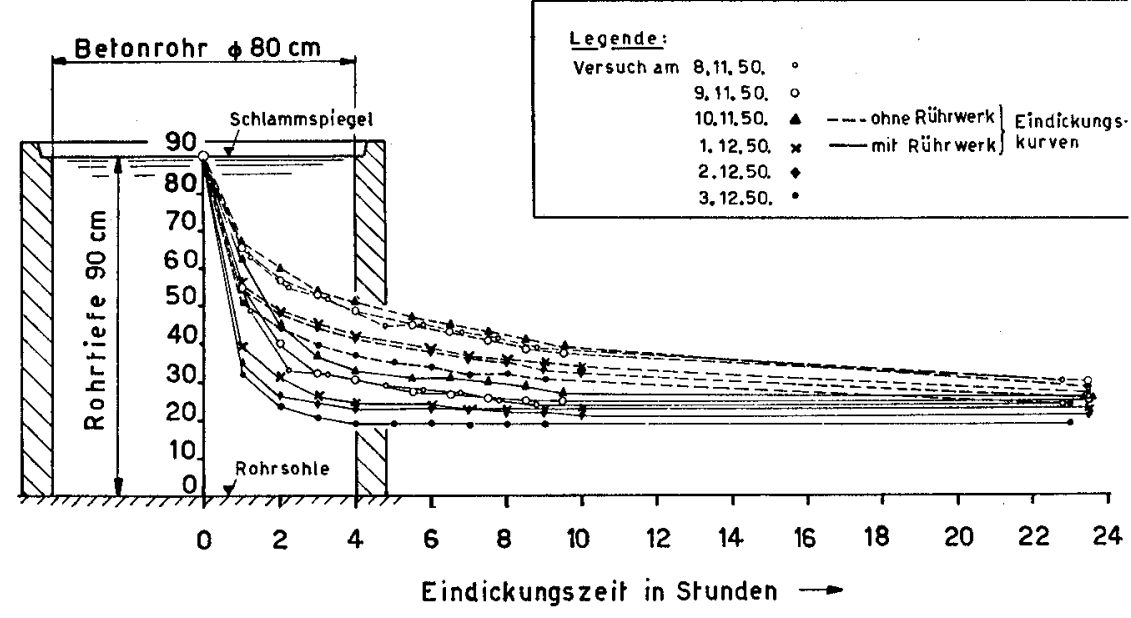

Wassergehalt: Ueberschussehlamm $=99,5-99,55 \%$

primärer Schlamm $=94,08-94,65 \%$

Abbildung 4

Eindickung von primärem Schlamm und Überschußschlamm aus Vollreinigung in äquivalenten Mengen.

mit Belebtschlamm allein. Hingegen war auch in dieser Versuchsserie nur eine Eindickung auf $1 / 3^{-1 / 4}$ des ursprünglichen Volumens möglich.

Dass trotzdem eine Volumenreduktion gegenüber einer Eindickung von Belebtschlamm allein erzielt werden kann, ergeben die nachfolgenden Überlegungen bzw. Berechnungen.

Nach Erstellung der biologischen Reinigung für die Stadt Zürich dürfte etwa mit folgendem Schlammanfall zu rechnen sein (pro Einwohner und Tag):

Tabelle 2

$g=$ Gramm $\quad E=$ Einwobner $; \quad T=$ Tag.

\begin{tabular}{|c|c|c|c|c|}
\hline & \multirow{2}{*}{$\begin{array}{c}\text { Wassergehalt } \\
\% \\
\text { (a) }\end{array}$} & \multicolumn{2}{|c|}{ Trockensubstanz } & \multirow{2}{*}{$\begin{array}{c}\text { Schlammanfall } \\
\text { l/E T } \\
\text { (d) }\end{array}$} \\
\hline & & $\begin{array}{l}\% \\
\text { (b) }\end{array}$ & $\begin{array}{l}\mathrm{g} / \mathrm{E} T \\
\text { (c) }\end{array}$ & \\
\hline $\begin{array}{l}\text { Primärer Schlamm } \\
\text { (sogenannter } \\
\text { Frischschlamm). . } \\
\text { Belebtschlamm } \\
\text { (U்berschuss- } \\
\text { schlamm). . . . }\end{array}$ & 94,0 & 0,5 & 48 & $\begin{array}{l}1,2=\frac{(c)}{(b)} \cdot \frac{1}{10} \\
9,6=\frac{(c)}{(b)} \cdot \frac{1}{10}\end{array}$ \\
\hline Total & 98,9 & $1,1=\frac{(\mathrm{c})}{(\mathrm{d})} \cdot \frac{1}{10}$ & 120 & $10,8=\frac{(c)}{(b)} \cdot \frac{1}{10}$ \\
\hline
\end{tabular}


Die Behälter wurden mit je 4001 Belebtschlamm und 501 primärem Schlamm, entsprechend dem Mischungsverhältnis 9,61/ET:I,2 1/ET, gefüllt und wie vorher die Schlammeindickung ohne und mit Rührwerk beobachtet (vgl. Abb.4). Der tatsächliche Wassergehalt vor dem Versuch betrug beim primären Schlamm 94,08-94,65\%, beim Belebtschlamm 99,5 bis $99,55 \%$.

Die Mittelwerte der sechs Untersuchungen sind in nachfolgender Tabelle 3 zusammengestellt, wobei $s^{1}$ ) wieder die Wurzel aus der Streuung

bedeutet:

$$
s^{2}=\frac{1}{N-1} \sum_{i=1}^{N}\left(x_{i}-\bar{x}\right)^{2}
$$

Tabelle 3

Eindickung auf Prozente des ursprünglichen Schlammvolumens (Mittelwerte)

\begin{tabular}{|c|c|c|c|c|c|c|}
\hline \multirow[t]{2}{*}{ Eindickungszeit } & \multicolumn{2}{|c|}{$4 \mathrm{~h}$} & \multicolumn{2}{|c|}{$8 \mathrm{~h}$} & \multicolumn{2}{|c|}{$24 \mathrm{~b}$} \\
\hline & \multicolumn{2}{|c|}{\begin{tabular}{c|c} 
mit $\mid$ ohne \\
Rührwerk
\end{tabular}} & \multicolumn{2}{|c|}{$\begin{array}{c}\text { mit I ohne } \\
\text { Rührwerk }\end{array}$} & \multicolumn{2}{|c|}{$\begin{array}{l}\text { mit | ohne } \\
\text { Rührwerk }\end{array}$} \\
\hline 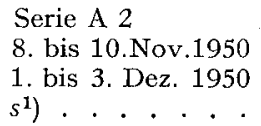 & $\begin{array}{r}29,9 \\
+\quad 6,2\end{array}$ & $\begin{array}{r}49,8 \\
+\quad 6,1\end{array}$ & $\begin{array}{r}26,8 \\
+\quad 4,3\end{array}$ & $\begin{array}{r}42,0 \\
+4,5\end{array}$ & $\begin{array}{r}25,6 \\
+\quad 2,9\end{array}$ & $\begin{array}{r}30,6 \\
+\quad 2,9\end{array}$ \\
\hline
\end{tabular}

1) Die Prozentzahlen von $s$ sind zu den Prozentzahlen des eingedickten Schlammes zu addieren bzw. von ihnen zu subtrahieren.

Aus einem Vergleich der Tabelle I mit der Tabelle 3 ist ersichtlich, dass sich nach einer 4stündigen Eindickungszeit mit Rührwerk das Schlammvolumen sowohl bei der Versuchsserie A I als auch bei A2 auf rund $30 \%$ reduzieren liess. Es stellt sich nun die Frage, ob es sich unter diesen Umständen überhaupt lohnt, den Frischschlamm zusammen mit dem Belebtschlamm einzudicken oder ob es nicht zweckmässiger wäre, nur den Belebtschlamm dem Eindickungsbecken zuzuführen. Folgende Überlegung gibt auf diese Frage Antwort:

Der Schlammanfall pro Einwohner (E) und Tag (T) beträgt auf Grund der Erhebungen in der Kläranlage Werdhölzli der Stadt Zürich (nach Tabelle 2) Io,8 l/ET mit I20 g/ET Trockensubstanz bei $98,9 \%$ Wassergehalt (WG). 
Wird gemäss Versuchsserie A I nur der Belebtschlamm allein eingedickt, so reduziert sich das gesamte Schlammvolumen gemäss Tabelle 4 auf $4, \mathrm{I} 1 / \mathrm{ET}$.

Tabelle 4

Scblammpolumen pro Einwobner und Tag

(uneingedickter Friscbscblamm und eingedickter Belebtscblamm)

\begin{tabular}{|c|c|}
\hline 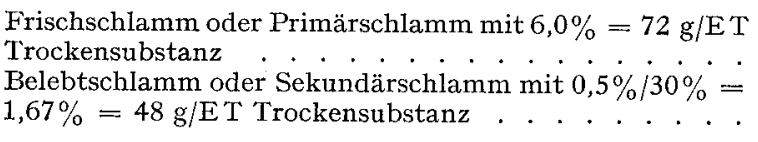 & $\begin{array}{l}1,21 / \mathrm{ET} \\
2,91 / \mathrm{ET}\end{array}$ \\
\hline Total als Mischschlamm: Mit 2,94\% bzw. 120 g/E T & $4,1 \mathrm{l} / \mathrm{E} \mathrm{T}$ \\
\hline
\end{tabular}

Falls nach Versuchsserie A2 Frischschlamm und Belebtschlamm zur Eindickung gelangen, reduziert sich das Schlammvolumen auf $30 \%$ von Io,8 1/ET $=3,251 / \mathrm{ET}$.

Das Volumen des gemischten Schlammes vor der Eindickung mit 98,9\% Wassergehalt und I0,8 1/ET Schlammvolumen (= I00\%) reduziert sich bei Eindickung des Belebtschlammes allein zu einem Schlamm von $97,06 \%$ Wassergehalt und. 4,I l/ET Volumen, das heisst auf $38 \%$ bzw. bei gemeinsamer Eindickung des gemischten Schlammes zu einem Schlamm von $96,3 \%$ Wassergehalt und $3,251 / \mathrm{ET}$, das heisst auf $30 \%$ seines ursprünglichen Volumens. Bei Eindickung des gemischten Schlammes gegenüber der Eindickung des Belebtschlammes allein kann somit eine Volumenverminderung auf $3,25 \mathrm{~S} / \mathrm{ET}$ statt $4, \mathrm{I} \mathrm{l} / \mathrm{ET}$ erzielt werden, obgleich in beiden Fällen eine Eindickung durch Rühren auf $30 \%$ des ursprünglichen Schlammvolumens möglich ist. Aus dieser Überlegung ergibt sich, dass stets der Primärschlamm mit dem Belebtschlamm gemischt eingedickt werden sollte, sofern beide Schlämme miteinander ausgefault oder einer andern, gemeinsamen Behandlung zugeführt werden sollen.

b) Versuchsserie $A 2^{\star}$. Etappenweise Eindickung: I. Etappe ohne Rühren, 2. Etappe mit Rühren.

Da die Eindickungsversuche zeigten, dass durch mechanische Eindikkung der Schlamm stets auf $1 / 3$ bis $1 / 4$ seines ursprünglichen Volumens eingedickt werden konnte, stellte sich die Frage, ob unter Umständen eine weitergehende Eindickung dadurch bewerkstelligt werden könnte, wenn zuerst der Schlamm während 8 Stunden einer normalen Schwerkrafteindickung ausgesetzt würde und erst dann eine zusätzliche Eindickung 


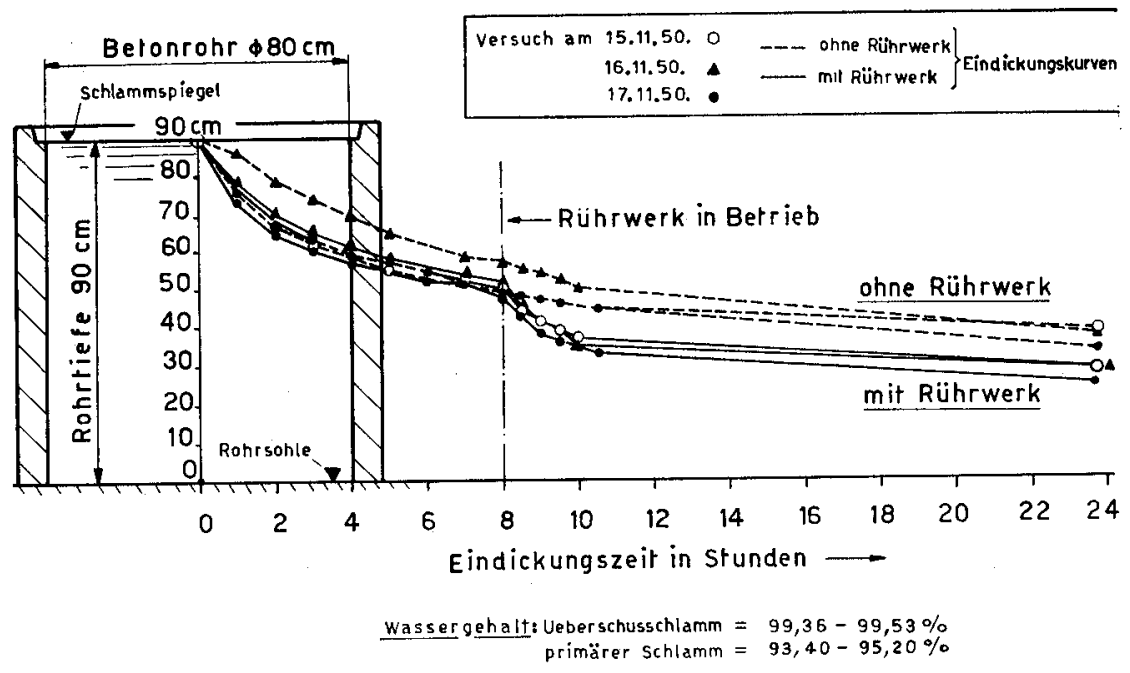

Abbildung 5

Eindickung von primärem Schlamm und ÜberschuBschlamm aus Vollreinigung in äquivalenten Mengen. Rührwerk erst 8 Stunden nach dem Schlammeinfüllen in Betrieb genommen.

durch das Rührwerk erfolgt. Das Ergebnis dieser Versuchsserie ist in Abbildung 5 dargestellt. Zur Eindickung gelangte wieder eine Mischung von je 4001 Úberschußschlamm aus der Vollreinigungsanlage mit 501 primärem Frischschlamm. Aus dem Verlauf der Eindickungskurven ist ersichtlich, dass während den ersten 8 Stunden sich an sämtlichen Tagen (mit Ausnahme des einen Versuches vom I6. November 1950) sämtliche Schlammspiegel angenähert gleichmässig absetzten. Die Wirkung des

Tabelle 5

Eindickung auf Prozente des ursprünglicben Scblammpolumens (Mittelmerte)

\begin{tabular}{|c|c|c|c|c|c|c|}
\hline \multirow[t]{2}{*}{ Eindickungszeit } & \multicolumn{2}{|c|}{$4 \mathrm{~h}$} & \multicolumn{2}{|c|}{$8 \mathrm{~h}$} & \multicolumn{2}{|c|}{$24 \mathrm{~h}$} \\
\hline & $\begin{array}{l}\text { mit } \\
\text { Rüh }\end{array}$ & $\begin{array}{l}\text { ohne } \\
\text { werk }\end{array}$ & $\begin{array}{l}\text { mit } \\
\text { Rii }\end{array}$ & $\begin{array}{l}\text { ohne } \\
\text { erk }\end{array}$ & $\begin{array}{l}\text { mit } \\
\mathrm{R} \ddot{u}\end{array}$ & $\begin{array}{l}\text { ohne } \\
\text { erk }\end{array}$ \\
\hline $\begin{array}{l}\text { Serie A } 2 \\
15 ., 16 . \text { und } \\
\text { 17. November } 1950 \\
\left.s^{1}\right) . . . . . .\end{array}$ & $\begin{array}{c}65,5 \\
\pm 2,5\end{array}$ & $\begin{array}{c}68,9 \\
\pm 6,5\end{array}$ & $\begin{array}{c}55,5 \\
\pm \quad 2,4\end{array}$ & $\begin{array}{l}57,9 \\
\pm \quad 4,4\end{array}$ & $\begin{array}{l}30,0 \\
\pm 2,0\end{array}$ & $\begin{array}{l}40,0 \\
\pm 3,0\end{array}$ \\
\hline
\end{tabular}

1) Die $s$-Werte in Prozent sind zu den Prozentzahlen des eingedickten Schlammes zu addieren bzw. von ihnen zu subtrahieren. 
Rührwerkeinsatzes nach 8 Stunden ist aus den Kurven deutlich ersichtlich. Der Wassergehalt des eingebrachten Überschußschlammes schwankte zwischen 99,36 und $99,53 \%$, derjenige des Primärschlammes zwischen 93,4 und $95,2 \%$. Die Untersuchungsergebnisse sind in Tabelle 5 als Mittelwerte zusammengestellt.

Aus der Versuchsserie $\mathrm{A} 2^{\star}$ ist ersichtlich, dass keine Vorteile zu erwarten sind, wenn vorerst der Schlamm im Eindickungsbehälter sich selbst überlassen und erst in einem späteren Zeitpunkt das Rührwerk in Gang gesetzt wird.

c) Versucbsserie A3. Belebtschlamm aus Vollreinigung $+2 / 3$ primärer Schlamm.

Zur Klarstellung des Einflusses der zum Belebtschlamm beigefügten Menge an primärem Schlamm für die Eindickung war geplant, in einer Versuchsserie $A_{3}$ zum Überschußschlamm aus der Vollreinigung (4I5 I) nur $2 / 3$ des gesamten Primärschlammes ( 35 l) beizumischen, in einer Versuchsserie A4 nur 1/3 des Primärschlammes. Da aus der Versuchsserie A3 und den bereits erwähnten Berechnungen hervorging, dass eine Mischung des Überschußschlammes mit der ganzen anfallenden Primärschlammenge am vorteilhaftesten ist, sofern eine gemeinsame Ausfaulung im Faulraum in Frage kommt, wurde auf die Durchführung der Versuchsserie A4 ver-

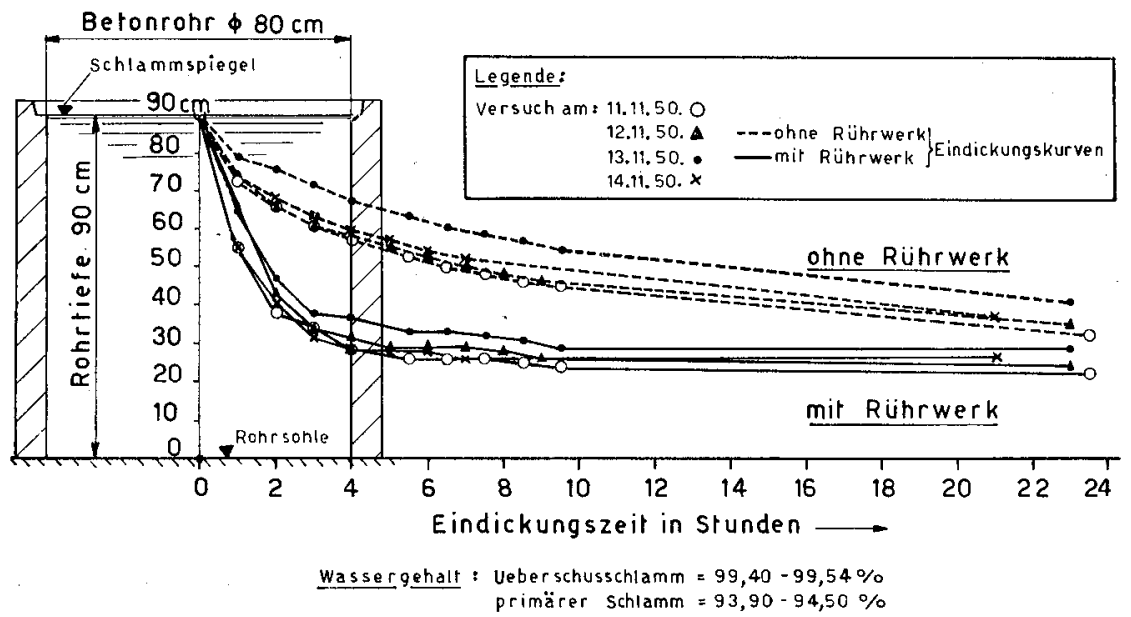

Abbildung 6

Eindickung einer reduzierten Menge (2/3) Primärschlamm und der vollen Menge Überschußschlamm aus Vollreinigung. 
zichtet. Trotzdem die Ergebnisse der Versuchsserie A 3 keine neuen Gesichtspunkte ergaben, sollen die Versuchsresultate nicht vorenthalten werden, da diese sehr eindeutig die Wirkung des Rührwerkes erkennen lassen (Abb.6). Der Wassergehalt des Überschußschlammes variierte zwischen 99,40 und $99,54 \%$, derjenige des Primärschlammes zwischen 93,9 und $94,5 \%$.

Über die Resultate der Mittelwerte der Eindickung samt Streuungen aus 4 Untersuchungspaaren gibt Tabelle 6 Auskunft.

Tabelle 6

Eindickung auf Prozente des ursprünglichen Schlammvolumens (Mittelmerte)

\begin{tabular}{|c|c|c|c|c|c|c|}
\hline \multirow[t]{2}{*}{ Eindickungszeit } & \multicolumn{2}{|c|}{$4 \mathrm{~h}$} & \multicolumn{2}{|c|}{$8 \mathrm{~h}$} & \multicolumn{2}{|c|}{$24 \mathrm{~h}$} \\
\hline & $\begin{array}{l}\text { mit } \\
\text { Rür }\end{array}$ & $\begin{array}{l}\text { ohne } \\
\text { erk }\end{array}$ & $\begin{array}{l}\text { mit } \\
\qquad \text { Rü }\end{array}$ & $\begin{array}{l}\text { ohne } \\
\text { erk }\end{array}$ & $\begin{array}{l}\text { mit } \\
\qquad \text { Rü }\end{array}$ & $\begin{array}{l}\text { ohne } \\
\text { erk }\end{array}$ \\
\hline $\begin{array}{l}\text { Serie A } 3 \\
\text { vom 11., } 12 ., 13 . \\
\text { und 14. Nov. } 1950 \\
\left.s^{1}\right) \quad . \quad . \quad . \quad . \quad .\end{array}$ & $\begin{array}{c}35,6 \\
\pm \quad 3,4\end{array}$ & $\begin{array}{c}67,4 \\
\pm 5,8\end{array}$ & $\begin{array}{l}30,6 \\
\pm 2,8\end{array}$ & $\begin{array}{r}56,1 \\
\pm \quad 5,3\end{array}$ & $\begin{array}{l}28,1 \\
\pm 3,4\end{array}$ & $\begin{array}{l}40,4 \\
\pm 4,2\end{array}$ \\
\hline
\end{tabular}

1) Die $s$-Werte in Prozent sind zu den Mittelwerten in Prozent zu addieren bzw. zu subtrahieren.

d) Versucbsserie A5. Belebtschlamm (Überschußschlamm) aus Vollreinigung + ausgefaulter Schlamm.

Aus der Überlegung heraus, dass ausgefaulter Schlamm bereits weitgehend mineralisiert ist und ein höheres spezifisches Gewicht aufweist als der Frischschlamm, wurden auch Versuche über die Eindickung von Belebtschlamm (4001) + ausgefaultem Schlamm (5o l) durchgeführt, in der Meinung, dass der schwere Faulschlamm eine vermehrte Eindickung des Belebtschlammes zur Folge haben könnte. Der Überschußschlamm wies für die drei Versuchsserien einen Wassergehalt von 99,13-99,42\% auf, der ausgefaulte Schlamm von $89,3-89,6 \%$. Das Resultat der Eindickungsversuche geht aus Abbildung 7 hervor. Daraus ist ersichtlich, dass wohl wieder ein Unterschied zwischen der Eindickung ohne und mit Rührwerk festgestellt werden konnte, dass jedoch die Schlammeindickung mit Rührwerk weniger rasch vor sich ging als bei der Zugabe von Frischschlamm. Zudem war die über dem Schlammspiegel stehende Flüssigkeit trübe und 


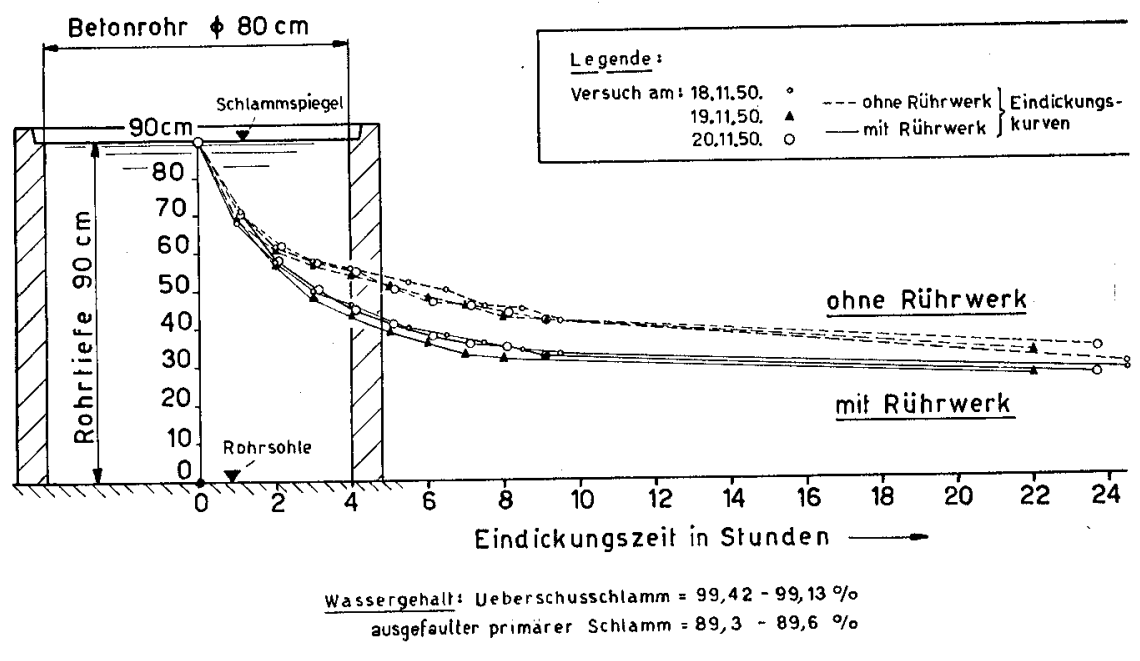

Abbildung 7

Eindickung von ausgefaultem Primärschlamm mit Überschußschlamm aus Vollreinigung.

mit Schwimmschlammfladen verschmutzt. Da zudem betriebstechnisch keine Vorteile zu erwarten wären - der ausgefaulte Schlamm müsste im Faulraum wieder aufgeheizt werden und würde nur zusätzliches Faulraumvolumen beanspruchen -, wurde auf die Weiterverfolgung dieser Versuche verzichtet. Es wird deshalb auch davon abgesehen, die Versuchsresultate einzeln in ausgewerteter Form wiederzugeben.

\section{Versuchsserie B}

Im Monat März I95I wurde eine weitere Versuchsserie durchgeführt. Der Schlamm, der damals als Überschußschlamm von der Versuchsanlage zur Verfügung stand, entstammte einem Teilreinigungsbetrieb. Am 2I. bzw. 22. März gelangte je ein Versuchspaar mit und ohne Rührwerkeindickung für den Teilreinigungsschlamm allein zur Durchführung, am 23. bzw. 24. März je ein Versuchspaar mit einem Mischschlamm, bestehend aus I Teil Frischschlamm und 8 Teilen Überschußschlamm. Der Frischschlamm oder primäre Schlamm wies einen Wassergehalt von 94,5 bis 94, $8 \%$ auf, der Überschußschlamm einen solchen von 99,33 bis $99,55 \%$. Trotzdem der Belebtschlamm bezüglich des Wassergehalts sich vom Vollreinigungsschlamm nicht unterschied, waren die Eindickungseffekte so- 
wohl mit als auch ohne Rührwerk überraschend gute, wie aus Abbildung 8 ersichtlich ist. Woher dies rührt, konnte nicht ermittelt werden.

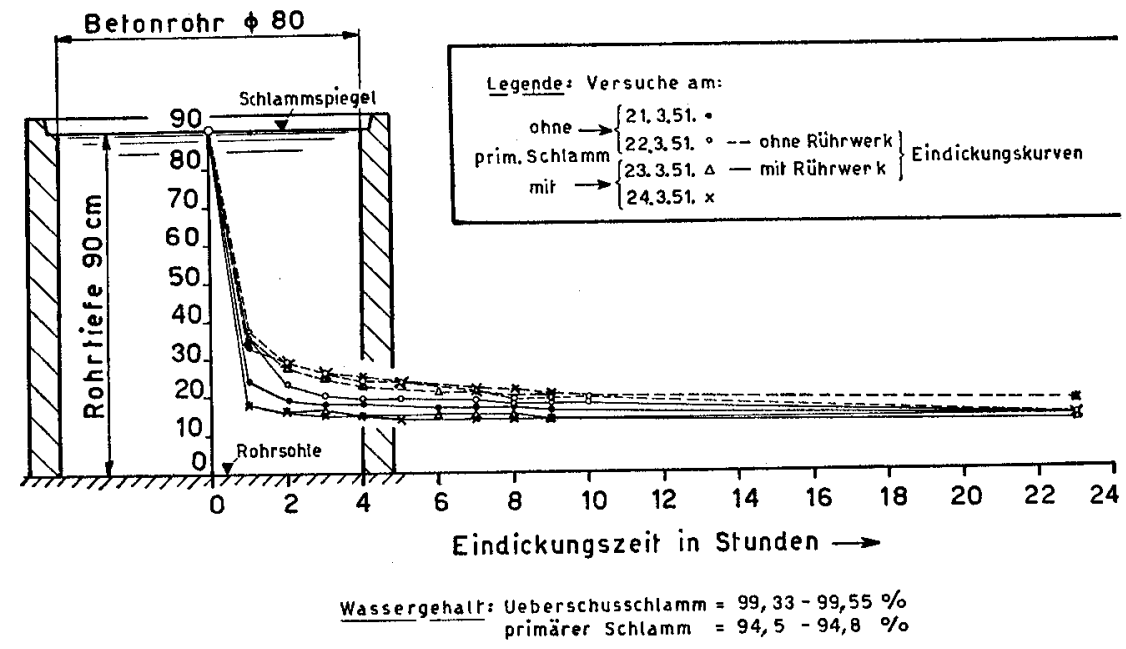

Abbildung 8

Eindickung von primärem Schlamm und Überschußschlamm aus Teilreinigung in äquivalenten Mengen.

Über die Mittelwerte der Eindickungsresultate samt den Streuungen geben die Tabellen $7 \mathrm{a}$ und $7 \mathrm{~b}$ Auskunft.

Tabelle $7 \mathrm{a}$

Eindickung des Überschußscblammes allein

Eindickung auf Proziente des ursprünglicben Scblammpolumens (Mittelmerte)

\begin{tabular}{|c|c|c|c|c|c|c|}
\hline \multirow[t]{2}{*}{ Eindickungszeit } & \multicolumn{2}{|c|}{$4 \mathrm{~h}$} & \multicolumn{2}{|c|}{$8 \mathrm{~h}$} & \multicolumn{2}{|c|}{$24 \mathrm{~h}$} \\
\hline & $\begin{array}{l}\text { mit } \\
\quad \text { Rül }\end{array}$ & $\begin{array}{l}\text { ohne } \\
\text { erk }\end{array}$ & $\begin{array}{l}\text { mit } \\
\quad \mathrm{Rü}\end{array}$ & $\begin{array}{l}\text { ohne } \\
\text { rk }\end{array}$ & $\begin{array}{r}\text { mit } \\
R\end{array}$ & $\begin{array}{l}\text { ohne } \\
\text { rk }\end{array}$ \\
\hline $\begin{array}{l}\text { Serie B1 } \\
\text { vom } 21 \text {. und } \\
\text { 22. März 1951 . } \\
s^{1} \text { ) . . . . . }\end{array}$ & $\begin{array}{r}20,6 \\
\pm 0,8\end{array}$ & $\begin{array}{r}26,7 \\
\pm 0\end{array}$ & $\begin{array}{r}19,4 \\
\pm 0,8\end{array}$ & $\begin{array}{l}21,1 \\
\pm 0\end{array}$ & $\begin{array}{l}15,6 \\
\pm 0\end{array}$ & $\begin{array}{l}15,6 \\
\pm 0\end{array}$ \\
\hline
\end{tabular}

1) Die $s$-Werte in Prozent sind zu den Mittelwerten in Prozent zu addieren bzw. zu subtrahieren. 
Tabelle $7 \mathrm{~b}$

Eindickung pon Frischschlamm und Überscbußschlamm

\begin{tabular}{|c|c|c|c|c|c|c|}
\hline \multirow[t]{2}{*}{ Eindickungszeit } & \multicolumn{2}{|c|}{$4 \mathrm{~h}$} & \multicolumn{2}{|c|}{$8 \mathrm{~h}$} & \multicolumn{2}{|c|}{$24 \mathrm{~h}$} \\
\hline & $\begin{array}{l}\text { mit } \\
\quad \text { Rül }\end{array}$ & $\begin{array}{l}\text { ohne } \\
\text { erk }\end{array}$ & $\begin{array}{l}\text { mit } \\
\text { Rü }\end{array}$ & $\begin{array}{l}\text { ohne } \\
\text { rk }\end{array}$ & $\begin{array}{l}\text { mit } \\
\quad \text { Rül }\end{array}$ & $\begin{array}{l}\text { ohne } \\
\text { rk }\end{array}$ \\
\hline $\begin{array}{l}\text { Serie B2 vom } 23 \text {. } \\
\text { und 24. März } 1951 \\
\text { s) } \mathbf{1} . . .\end{array}$ & $\begin{array}{c}16,7 \\
\pm 0,0\end{array}$ & $\begin{array}{r}26,7 \\
\pm 1,6\end{array}$ & $\begin{array}{l}16,1 \\
\pm 0,8\end{array}$ & $\begin{array}{l}22,8 \\
\pm 0,8\end{array}$ & $\begin{array}{r}15,0 \\
\pm 0,8\end{array}$ & $\begin{array}{l}20 \% \\
\pm 0,0\end{array}$ \\
\hline
\end{tabular}

1) Die s-Werte in Prozent sind zu den Mittelwerten in Prozent zu addieren bzw. zu subtrahieren.

Aus den Tabellen $7 \mathrm{a}$ und $7 \mathrm{~b}$ ist ersichtlich, dass die Konzentrierung des Schlammes mit Rührwerk wiederum eine raschere Eindickung gewährleistet, als dies ohne Rührwerk der Fall ist. Zudem wurde bei obigen Versuchen mit Zugabe von Frischschlamm und Rührwerk eine vermehrte Eindickung des Frischschlammes (Frischschlamm + Belebtschlamm) gegenüber dem Belebtschlamm allein festgestellt.

Führen wir dieselbe Berechnung durch, wie sie für die Versuchsserie A2 erfolgt ist, so ergibt sich, dass bei einem Mischschlamm ohne Eindickung I,2 1 Frischschlamm $+9,61$ Belebtschlamm $=10,81 / \mathrm{ET}=100 \%$ Schlamm entsteht, während bei Eindickung des Belebtschlammes allein auf rund $20 \%$ nach 8 Stunden und Zugabe des eingedickten Überschussschlammes zum uneingedickten Frischschlamm noch mit einem Volumen von $3, \mathrm{I} 21 \mathrm{Schlamm} / \mathrm{ET}=28,8 \%$ gerechnet werden muss, gegenüber einem Volumen von 2,I61 Schlamm/ET $=20,0 \%$ beim Eindicken beider Schlämme zusammen. Es zeigt sich somit, dass die gemeinsame Eindickung von Frischschlamm + Belebtschlamm auch in diesem Falle vorteilhafter ist als die Eindickung des Belebtschlammes allein.

\section{Versuchsserie C}

Aus dem Taschenbuch von ImHoFf (I5.Auflage, S.165) geht hervor, dass zur Eindickung von Schlamm Zusätze von Chlor vorteilhaft sind, und zwar 0,2 bis $0,5 \mathrm{~g} / \mathrm{ET}$. Um die Wirkung der Chlorzusätze auszuprobieren, wurden Parallelversuche mit Eindickung von Überschußschlamm allein (ohne Anwendung eines Rührwerkes) mit und ohne Chlorzusatz durch- 


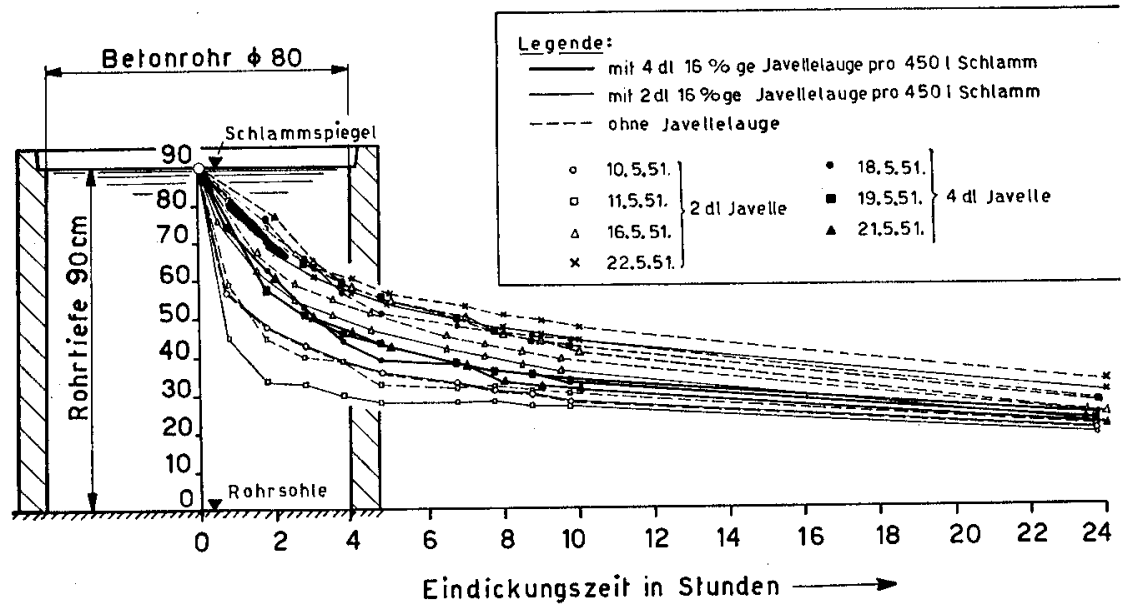

Wassergehalt des Ueberschusschlammes: $99,5-99,6 \%$

Abbildung 9

Eindickung von Überschußschlamm aus Vollreinigung ohne und mit Chlorzugabe (Riihrwerk ausser Betrieb).

geführt. Die Chlorzugabe erfolgte durch Zusatz von Javellauge mit zwei verschiedenen Dosierungen, nämlich entsprechend einer Chlorzugabe von 0,7 bzw. I,4 g/ET.

Die Ergebnisse der Schlammeindickungsversuche gehen aus der Abbildung 9 hervor. In den Tabellen $8 \mathrm{a}$ und $8 \mathrm{~b}$ sind die Mittelwerte der Versuchsergebnisse samt den Streuungen für eine Chlorzugabe von 0,7 bzw. I,4 g/ET aufgeführt.

Tabelle 8 a

Cblorzugabe $=0,7 \mathrm{~g} / \mathrm{E} \mathrm{T} \mathrm{bzw.71} \mathrm{g} / \mathrm{m}^{3}$ Überscbußscblamm

Eindickung auf Prozente des ursprünglicben Scblammvolumens (Mittelmerte)

\begin{tabular}{|c|c|c|c|c|c|c|}
\hline \multirow[t]{2}{*}{ Eindickungszeit } & \multicolumn{2}{|c|}{$4 \mathrm{~h}$} & \multicolumn{2}{|c|}{$8 \mathrm{~h}$} & \multicolumn{2}{|c|}{$24 \mathrm{~h}$} \\
\hline & $\begin{array}{l}\text { mit } \\
\text { Chlor }\end{array}$ & $\begin{array}{l}\text { ohne } \\
\text { lgabe }\end{array}$ & $\begin{array}{l}\text { mit } \\
\text { Chlo }\end{array}$ & $\begin{array}{l}\text { ohne } \\
\text { gabe }\end{array}$ & $\begin{array}{l}\text { mit } \\
\text { Chlo }\end{array}$ & $\begin{array}{l}\text { ohne } \\
\text { gabe }\end{array}$ \\
\hline 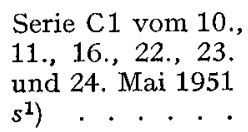 & $\begin{array}{l}54,0 \\
\pm 13,0\end{array}$ & $\begin{array}{r}58,5 \\
+\quad 12,2\end{array}$ & $\begin{array}{r}44,6 \\
\pm 9,8\end{array}$ & $\begin{array}{c}47,4 \\
\pm 10,0\end{array}$ & $\begin{array}{l}28,4 \\
\pm 5,8\end{array}$ & $\begin{array}{c}30,6 \\
\pm 7,0\end{array}$ \\
\hline
\end{tabular}

1) Die $s$-Werte in Prozent sind zu den Mittelwerten in Prozent zu addieren bzw. zu subtrahieren. 


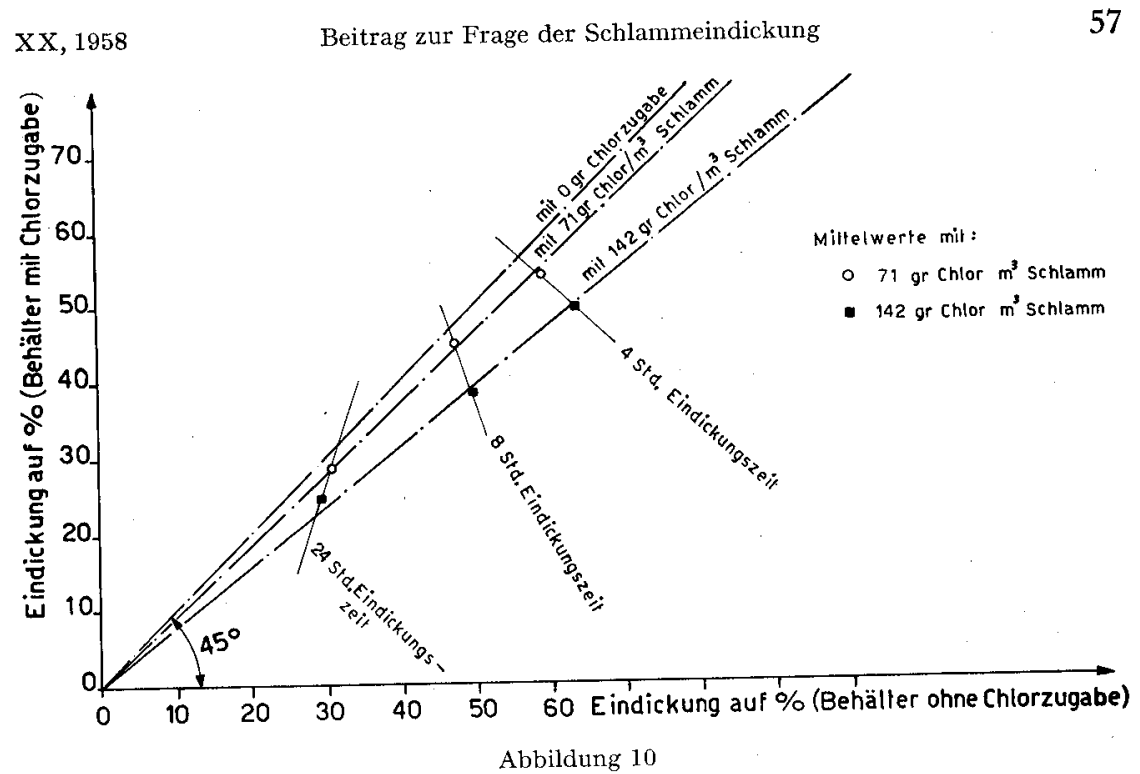

Einwirkung verschiedener Chlorzugaben auf die Eindickung von belebtem Schlamm (Parallelversuche).

Tabelle $8 \mathrm{~b}$

Eindickung auf Prozent des ursprünglicben Scblammolumens (Mittelperte) Cblorzugabe $=\mathrm{I}, 4 \mathrm{~g} / \mathrm{E} \mathrm{T}$ bezw. $\mathrm{I}_{42} \mathrm{~g} / \mathrm{m}^{3}$ Überscbußscblamm

\begin{tabular}{|c|c|c|c|c|c|c|}
\hline \multirow{3}{*}{ 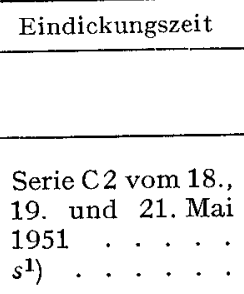 } & \multicolumn{2}{|c|}{$4 \mathrm{~h}$} & \multicolumn{2}{|c|}{$8 \mathrm{~h}$} & \multicolumn{2}{|c|}{$24 \mathrm{~h}$} \\
\hline & \multicolumn{2}{|c|}{$\begin{array}{l}\text { mit ohne } \\
\text { Chlorzugabe }\end{array}$} & \multicolumn{2}{|c|}{$\begin{array}{l}\text { mit | ohne } \\
\text { Chlorzugabe }\end{array}$} & \multicolumn{2}{|c|}{$\begin{array}{l}\text { mit | ohne } \\
\text { Chlorzugabe }\end{array}$} \\
\hline & $\begin{array}{l}49,6 \\
+1,7\end{array}$ & $\begin{array}{l}63,6 \\
\pm 1,3\end{array}$ & $\begin{array}{l}37,8 \\
\pm 1,9\end{array}$ & $\begin{array}{r}49,6 \\
\pm 1,7\end{array}$ & $\begin{array}{l}24,5 \\
\pm 2,2\end{array}$ & $\begin{array}{l}29,3 \\
\pm 2,2\end{array}$ \\
\hline
\end{tabular}

Diese Mittelwerte sind in Abbildung ro graphisch dargestellt. Daraus geht hervor, dass in vorliegendem Falle die Schlammeindickung um so besser bzw. rascher vor sich ging, je grösser die Chlorzugabe bemessen wurde. Andere Verbesserungen durch Chlorzugabe konnten nicht festgestellt werden. Da die Verbesserung der Eindickungswirkung durch normale Chlorzugabe für die in Zürich vorliegenden Verhältnisse nicht über- 
zeugend ausfiel, so wurde im Erweiterungsprojekt für die Kläranlage Werdhölzli der Stadt Zürich auf eine Chlorzugabe verzichtet.

\section{Schlussfolgerungen}

I. Eine Eindickung des sehr wasserhaltigen Überschußschlammes aus Belebtschlammanlagen vor dem Pumpen in die Faulräume ist erforderlich, um an Faulraumvolumen und an Faulgas zur Heizung der Faulräume zu sparen.

2. Eine Eindickung des Überschußschlammes kann durch ruhiges Absetzenlassen im Vorklärbecken bzw. in Behältern erfolgen oder in speziellen Eindickungsbecken mit maschinellem Umrühren. Mit beiden Verfahren erreicht man Eindickungseffekte. Die Eindickung geht mit Rührwerken rascher vor sich. Der praktische Eindickungsvorgang ist statt in 24 Stunden ohne Rührwerk in rund 3-4 Stunden mit Rührwerk beendet. Die Eindickung mit Rührwerken geht zudem gleichmässiger vor sich als ohne. Die Streuungen der einzelnen Versuchsresultate sind geringer.

3. Es ist denkbar, dass nur der Überschußschlamm allein eingedickt und dieser, zusammen mit dem Frischschlamm der Vorklärbecken gemischt, in den Faulraum geführt wird. Auf Grund vorstehender Versuche ist es jedoch zweckmässiger, die beiden Schlämme zu mischen und gemeinsam einzudicken.

4. Die Schlammeindickung gehorcht gleichen Gesetzen wie die Setzung von Gebäuden über Tonschichten.

5. Mit einer etappenweisen Eindickung (zuerst ruhiges Stehenlassen des Schlammes und nachherige Inbetriebsetzung des Rührwerkes) können keine besseren Eindickungsergebnisse erzielt werden.

6. Die Zugabe von ausgefaultem Schlamm zum Überschußschlamm zwecks gemeinsamer Eindickung der beiden Schlämme bietet keinerlei Vorteile und ist in betriebstechnischer und ästhetischer Hinsicht abzulehnen.

7. Mit Zugabe von Chlor zum Schlamm konnte eine bessere Eindickung erzielt werden. Die Chlorzugabe erwies sich jedoch bei den vorgenommenen Versuchen für den in Zürich anfallenden Schlamm nicht als notwendig. Es ist wirtschaftlicher, etwas länger maschinell einzudicken, als Chlor zuzusetzen. 
8. Der Überschußschlamm wies einen konstanten Wassergehalt von $99,5 \%$ auf. Praktisch liess sich nur ein Wassergehalt von 97 bis $98 \%$ nach der Eindickung erzielen.

9. Es ist nicht ausgeschlossen, dass sich in tieferen Eindickungsbecken (Schlammtiefen in den Versuchsbecken $=90 \mathrm{~cm}$ ) bessere Eindickungsergebnisse erzielen lassen. Diesbezügliche Untersuchungen sind in Aussicht genommen.

Io. Die Umdrehungsgeschwindigkeit des Rührwerkes wurde während sämtlicher Versuche konstant gehalten ( I U/min). Über den Einfluss einer Änderung der Umdrehungsgeschwindigkeiten kann auf Grund der Versuche keine Aussage gemacht werden.

II. Die Beschickung der Eindickungsbecken erfolgte chargenweise. Eine Eindickung im Durchlaufbetrieb wurde nicht vorgenommen.

Es ist mir ein Bedürfnis, den Herren K. BRÄM, Betriebsleiter, und FR. JÜNI, Klärmeister der Kläranlagen der Stadt Zürich, für die Beratung bei der Versuchsanordnung sowie für die grosse Arbeit beim Aufbau und während der Durchführung sämtlicher Versuche bestens zu danken. 